\title{
Homemade heroin substitute causing hallucinations
}

Desomorphine, a synthetic opioid, can be synthesised from codeine, red phosphorous and iodine, however, the end result is impure and highly corrosive. ${ }^{1}$ The street name is 'krokodil' due to the characteristic skin scales users have after use. The effects on the user are similar to that of heroin, although they are reduced from 4-8 hours for heroin to 1 hour for homemade desomorphine. ${ }^{2}$ Due to the impure nature of krokodil, phlebitis and gangrene are common. Tissue damage is drastic and it is believed that users' life expectancy is only $2-3$ years. $^{2}$

$A B$, a 19 year old female sex worker, presented after having lived in the United Kingdom for 27 months. She came to the UK illegally from Romania to be with her boyfriend who was already working in the country. She lives in several different locations, depending on where she is needed to work. Informed consent for publication of case material was provided.

AB had been taking drugs since her arrival in the UK, to help with the 'pain of having no money', and being forced 'to do horrible things to be able to afford to eat'. She felt her addiction was getting worse, and she that it needed to stop as it was costing too much to keep her addiction at bay. The pattern was of frequent use, injecting $£ 80$ worth of heroin daily. Recently, she had been unable to afford this, and had been using a cheaper option available to her. Without heroin she suffers a painful stomach and becomes cold. Since using the cheaper option, she has experienced hallucinations and skin changes. AB has also used amphetamines occasionally when heroin is not readily available, but had not used it in the 3 months prior to presentation.

AB normally contacted relatives at home twice a week, however, she hadn't done so in the preceding two weeks. AB had regular, transient thoughts of self-harm since arriving in the UK.

AB looked very thin, she was seated and leaning on her boyfriend throughout the meeting. Her skin was very pale, and there was a 'damp' smell coming from her. Her blood pressure was 126/72 mmHg and heart rate was 87 beats per minute. She had puncture wounds bilaterally in her antecubital fossae. She had three very large scabs each 4-5 cms in diameter on her face. She had 'popped' skin lesions surrounding hypodermic puncture sites in bilateral antecubital fossae. Her hands were erythematous and swollen, with puncture wounds from attempted and successful injections. AB suffered from poor hygiene; all

Correspondence

MrT Lemon

email: lemonti@cf.ac.uk teeth appeared present, although she had noticed some recent tooth discolouration. Her clothes were not clean but were appropriate for weather and location. No abnormal involuntary movements were noted, but she had a slumped posture and was supported by her boyfriend.

AB was polite throughout the consultation, using appropriate salutations and titles. She had a reduced level of eye contact, and did not build rapport with the history taker. She was attentive and not easily distracted. AB was a foreign speaker, with a moderate to good level English and spoke at normal volume, slower than normal rate, and normal tone. Sentences were brief, and sometime monosyllabic, although fluency was as may be expected by a non-native speaker. There was no flight of ideas or neologisms.

Importantly, this case suggests homemade Desomorphine may cause hallucinations, unlike heroin use which is, anecdotally, believed to 'protect' against visual hallucinations. AB was suffering visual hallucinations that coincided with the commencement of taking the cheaper heroin like substance. The dermatological lesions on the face and the antecubital fossae are typical of the corrosive action of homemade desomorphine. This case highlights the importance of looking for non-typical symptoms in substance abusers.

Arguably, this is the first case of homemade Desomorphine use in the United Kingdom (street name 'krokodil'), suggesting that some dealers are now producing a cheaper option to heroin. This is surprising, as Desomorphine arose in Russia due to the easy access of over the counter codeine, which is not the case in the UK. This raises the question as to whether homemade Desomorphine is being imported into, or produced within, the United Kingdom. It is also an example of the common presentations of homemade Desomorphine use - namely hallucinations and skin lesions.

TI Lemon

School of Medicine, Cardiff University, University Hospital of Wales, Cardiff, Wales, United Kingdom

\section{References}

1. Savchuk S, Barsegyan S, Barsegyan B Kolesov G. Chromatographic study of expert and biological samples containing desomorphine. Journal of Analytical Chemistry 2011; 63 (4): 361-70.

2. Skowronek R, Celi ski R, Chowaniec C. Crocodile - new dangerous designer drug of abuse from the East. Clinical Toxicology 2012; 50 (4): 269. 